\title{
Overview on Monitoring of Therapeutic Drugs
}

\author{
Shivaji Prataprao Gawade \\ Sahyadri College of Pharmacy, Methwade, Tal.Sangola, Dist.Solapur-413307,M.S. INDIA.
}

\begin{abstract}
Purpose: The drug monitoring services are to obtain clinical pharmacokinetic data that results into fewer adverse reactions, shorter intensive care and shortened overall period of hospitalization. The monitoring of plasma level of therapeutic drugs can help to distinguish between noncompliant and a true non responder patient and to individualize dosage regimen in a particular disease state. Methods: The drug monitoring measurements involve interpretation of number of variables such as date, time, route of administration, clinical state of patient. Methods include, three components structural, process and output measurement, three types of drug monitoring, clinical, biochemical and plasma drug concentration measurements; analytical methods for quantification and modern highly sensitive methods used for concentration measurement from micro volume samples. Conclusion: The monitoring of therapeutic drugs is especially for personalizing medicine in ageing population and to optimize the efficacy of drugs in children as there are differences in absorption, disposition and pharmacological effects of most drugs between adult and children. The present overview, exemplifies various parameters influencing drug monitoring, priori and posteriori drug monitoring, limitations and applications of drug monitoring.
\end{abstract}

Key words: Therapeutic range, Priori, Posteriori, Sampling, Monitoring.

\section{INTRODUCTION}

The monitoring of therapeutic drugs is a segment of clinical pharmacy or clinical pharmacology that deals with drugs or drug metabolite concentration in the blood. The monitoring is performed because, as the dosage is increased from low dose to high dose, toxic manifestations are encountered which may be due to high dose for the patient. The response varies for the same dose based on patient status and disease condition. The outcome of measurement may be desirable or undesirable for the patient or it may leads to intolerable or therapeutic implications. The drug monitoring will assist physician to individualize dosage regimen of the patient that is influenced by various factors based on patient's history such as age, diet, smoking and disease conditions such as functions of organs such as kidney, liver, heart and thyroid gland. The dosage individualization will produce target concentration for preventing certain disease conditions. e.g. Phenytoin to prevent seizures after neurosurgery or trauma, Lithium dosage to prevent manic depressive attacks,
Cyclosporine dosage to prevent transplant rejection. ${ }^{1}$ The drug monitoring is used in two major situations. First is prophylactic use to maintain absence of clinical conditions like seizures, cardiac arrhythmia, depressive or maniac disorder, relapse from asthmatic episodes and organ rejection the second use is to avoid serious toxicity such as nephrotoxicity with amino glycoside antibiotics. The drug monitoring is based on two principles. There is graded dose response relationship with most of the drugs used for monitoring and that the therapeutic response of the drug, do not suddenly switch off from the lower limit or toxic response or do not suddenly appear at the upper limit of therapeutic range.

As the drug is given to maintain therapeutic level in circulation, it is given at regular interval of plasma $t \frac{1}{2}$ of the drug for the measurement during steady state. The information regarding pharmacokinetic parameters of the drug is used to retrieve the changes in the dosage regimen. The change in pharmacokinetic parameters may be due
DOI: 10.5530/ijopp.9.3.4

Address for correspondence: Dr Shivaji Prataprao Gawade,

Principal and Professor Sahyadri College of Pharmacy, Methwade

Tal.Sangola, Dist. Solapur-413307, M.S., INDIA. E-mail:gneskw@gmail.com

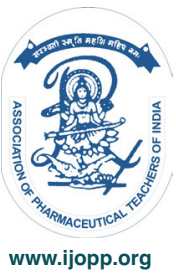


to changes in the physiological state of the patient, change in the diet or administration of additional drug that will influence the dosage regimen. Therapeutic range and sampling time are the critical parameters for monitoring of therapeutic drugs.

\section{THERAPEUTIC RANGE}

Therapeutic range is the range of serum concentration for which most of the patient will effectively respond with minimum side effects. The clinical response in this range is relatively high with minimum adverse effects. Therapeutic range is influenced by the extent of the drug bound to protein and unbound form of the drug, disease state of the individual, age of the patient, the drug interactions when patient on combination therapy and sensitivity of the method used for the drug concentration measurements. The therapeutic range is also affected by in vitro mixing of drug in combination therapy e.g. mixing of intravenous solution of amino glycoside antibiotics with penicillin will cause degradation. Sometimes active metabolite of the drug is used to assess the therapeutic range of the parent drug e.g. use of n-acetyl procainamide (NAPA) is used instead of procainamide for the drug concentration measurement. Administrations of digoxin generate digoxin like immune reactive substance (DLIS) that mimic digoxin in neonates.

\section{SAMPLING TIME}

Information on patient's specific pharmacokinetic parameters can be obtained by sampling early after the drug therapy is initiated. The patient specific pharmacokinetic parameters can be obtained by manipulating and specifying the dosage regimen. The steady state values can be obtained by relating serum level to a target concentration range.

\section{Factors influencing sampling time}

i) If the sampling is carried out too early shortly after the dose, the information on status of drug and drug clearance will be missing. ii) If sampling is delayed for potentially toxic drugs until steady state is attained, it may cause damage to the organ. e.g Gentamicin is administered in clinical conditions such as edema, ascites. At steady state the plasma concentration is higher than $90 \%$. The next dose is reduced by half and sampling is performed after 2 half lives. If the target concentration is not achieved with this dose, the next dose is reduced by half and sampling is performed after 2 half lives.(iii) Distribution kinetics a)If the drug is absorbed orally in first 2 hours after dosing, sampling is performed 2 hours of the oral dose. Food intake, posture and physical state of patient during sampling affects the absorption. b) If the drug is rapidly distributed e.g gentamicin, kanamycin, sampling is performed 2 hours of the oral dosing. If the distribution of drug is slow, sampling of digoxin is performed 6 hours after the dose and lithium sampling is performed just before the last dose. (iv) If the drug is given with constant maintenance dose without loading dose, the dosing is performed at regular interval, the sampling time in this situation will be at 2 half-lives'. (v) Peak and Trough level rather than dosing interval, is used to evaluate the dosage of drugs with short $t^{1 / 2}$ than the dosing interval e.g. gentamycin. ${ }^{2}$

\section{CHARACTERISTIC OF THERAPEUTIC DRUGS APPLICABLE FOR MONITORING}

(i) Monitoring of therapeutic drugs is based on the observable clinical situation i.e. end point of the drug. The end point could be therapeutic, toxic or interm diate, e.g. Prevention of stroke, pulmonary embolism, seizure episodes are therapeutic end points; kidney dysfunction, arrhythmia are toxic end points whereas prothrombin time are intermediate end points. When clearly observable therapeutic or toxic end points are absent, practicable intermediate end point may be used. The plasma digoxin concentration measurements in patients on digoxin therapy, who are with border line renal function, geriatrics or patient with rapid atrial fibrillation, require higher doses of digoxin to restore the normal heart rate. ${ }^{3}$ (ii) Drugs with narrow therapeutic range are used for the monitoring. These drugs have therapeutic index. 2-3. Therapeutic index. is the ratio of top to bottom serum concentration within the therapeutic range. (iii) Drugs with unpredictable $\mathrm{PK} / \mathrm{PD}$ relationship: The dose of drug producing sub therapeutic response in one patient, yield toxic effect in another patient. The PD indices include plasma lipid level, blood glucose, blood pressure, plasma clotting time etc which provides relationship between dose and plasma or blood drug concentration and pharmacodynamic effects. There is also wide inter patient variability in PK parameters, such as absorption, distribution, metabolism and excretion. There are differences in PK and PD of most drugs between adults and children. In children, sampling volume is limited. Therefore highly sensitive analytical methods are required for the drug sample measurements. ${ }^{4}$ (iv) The therapeutic drug used for monitoring could be either toxic or ineffective that can render the patient in a great risk. (v), Acute, short term or intermittent dosing of the drug, producing variable effects, are not well regulated with the plasma concentration of the drug. Sometimes pharmacological effects of the drug persist for a longer period of time. (vi), The relationship between plasma concentration of drug and therapeutic 
effect is stronger than that of dose of the drug administered and it's therapeutic effect. ${ }^{5}$ (vii) Plasma concentration measurements of drug should be at appropriate timings at regular intervals. Measurements at inappropriate time intervals will be misuse of the method. ${ }^{6}$ (viii) Use of accurate, precise, specific and sensitive assay methods for drug concentration measurement in the biological fluid is sin qui non of drug monitoring.

\section{METHODS USED FOR MONITORING OF THERAPEUTIC DRUGS}

The structural components of monitoring are equipments used to test therapeutic monitoring, qualification of the clinical and laboratory staff, supervisor and administrative organization. The process component of the monitoring measurements include, appropriate indications of serum drug levels, timing of sample collections, communication of results to the clinician and monitoring for appropriate clinician response to the treatment recommendation and patient response to the treatment and the output measurement components include assessment of drug induced adverse reactions, cure rates, mortality rates and cost saving associated with the monitoring. ${ }^{7}$

Plasma or serum samples are usually used however, for the drugs like cyclosporine whole blood is used as there is a large shift of drug between red blood cells and plasma with the change in temperature and storage.

Three different indicators are used for drug monitoring: (i) monitoring from the clinical effects such as lowering of blood glucose, prophylaxis of migraine and reduction in inflammation. (ii) Monitoring of biochemical effects preceding the biological effect such as glucose modulation by insulin. An anti-inflammatory effect preceding with lowering of CRP (C- reactive protein) level. (iii) Plasma drug concentration measurements and its implications to biological therapeutic effects. e.g. cardiac arrhythmia by digoxin, seizures by phenytoin.

\section{Analytical methods for monitoring of therapeutic drugs}

Spectrophotometry and Fluorometry : The level of sensitivity of these methods is in $\mu \mathrm{g} / \mathrm{ml}$ range.

Thin layer chromatography : It is used to identify and quantify the drug. However it is time consuming and less sensitive.

HPLC and GLS : This method is highly sensitive, precise and specific. However it requires Extraction and Column degeneration that occurs with time. HPLC is more preferred to GLC.

Radioimmunoassay (RIA) :This method is sensitive and precise however, it requires radio nucleotide.
Enzyme linked immunoabsorbant assay (ELISA): Unlike RIA, in ELISA radioactive trace is not required.

Fluorescence polarization immunoassay (FPIA): FPIA combines competitive protein binding with fluorescence polarization to give direct measurement without requirement of separation. ${ }^{8}$

\section{DOSAGE ADJUSTMENNT DUE TO UNEXPECTED SERUM CONCENTRATION OF DRUG ${ }^{8}$}

The causes responsible for variability in serum concentration are noncompliance, inappropriate dosing, malabsorption, inadequate bioavailabilty and multidrug regimen, altered protein binding in patients of hepatic and renal diseases. The newly adjusted dose can be obtained from drugs with linear pharmacokinetics.

Adjusted new dose $=$ usual dose $\mathrm{X}$ desired drug concentration/ usual dose concentration

\section{Modern methods for monitoring of therapeutic drugs}

Modern methods used for monitoring of therapeutic drugs are highly sensitive, ultra rapid, uses micro volume and requires highly expensive sophisticated instrumentation such as Liquid chromatography high resolution TOF mass spectrometry, LC/MS/MS small volume micro assay technique, paper spray mass spectrometry etc. A liquid chromatography high resolution TOF mass spectrometry (LC-HRMS) was used to detect Atenolol by accurate mass to charge ratio of the target analyte. Micro volume of $30 \mu \mathrm{l}$ of blood sample was required for analysis. ${ }^{9}$ Micro assay technique of small volume sample measurements from pediatric and neonatal patient's samples using modern detection system LC/MS/MS for improved detection have been used. Inborn errors of metabolism using Guthric cards were detected from small volume dried blood spot samples by this microassay. ${ }^{10}$ Direct online injection method reduced sample preparation step, handing requirements of infectious bioactive substances, improves reproducibility and minimizes sample manipulation and contamination. The automated methods for liquid chromatography include restricted access materials, turbulent flow chromatography, online solid phase extraction methods such as molecularly imprinted polymers, in tube solid phase micro extraction and packed syringe micro extraction for extraction of specific analyte from complex mixture of substances. ${ }^{11}$ The paper spray mass spectrometry was used to generate gas phase ions directly from the blood card paper that stores dried blood samples. The entire time of preparation and analysis of blood samples is about 30 seconds. The concentration of drug is detected quantitatively in the range of $1 \mathrm{ng} / \mathrm{ml}^{12}$

Indian Journal of Pharmacy Practice, Vol 9, Issue 3, Jul-Sep, 2016 


\section{QUALITY ASSURANCE, CRITERIA OF EVALUATION AND COMPLIANCE OF DRUG MONITORING}

\section{Quality assurance}

Quality assurance of drug monitoring involves correct timing, drawing of blood samples, reporting of drug concentration, measurement consultation with senior of the team and patient compliance.

\section{Criteria of evaluation}

The criteria of evaluation of drug monitoring are appropriateness in performing the concentration measurements, accuracy and completeness of information provided, appropriateness in action taken on the results, improvement in scheduling, drawing and reporting and appropriateness in documentation which includes, plasma drug concentration measurement, times at which the last dose was administered and the time at which blood samples were taken.

\section{Factors influencing compliance}

Factors influencing compliance of drug monitoring are (i) Variation in plasma concentration in disease conditions. (ii) Prediction of over dosage and under dosage from plasma concentration. e.g. low dose, impaired function of small bowel and low drug clearance: In renal failure, clearance is affected whereas in hepatic failure, clearance remains unaffected.(iii) Increased tissue binding will decrease the plasma concentration thereby increase in the volume of distribution ( $\mathrm{Vd}$ ) e.g. chloroquine. ii) In obese individuals: Digoxin cannot enter into the fatty tissue with consequent decrease in the $\mathrm{Vd}$.

\section{INDICATIONS AND BENEFITS OF DRUG MONITORING}

\section{Indications}

(i) Monitoring of therapeutic drugs is indicated when the relationship between plasma drug concentration and pharmacologic effect is experimentally proven. (ii) It provides information of blood drug concentration that influences medication management e.g. In patient of renal failure, the dosage regimen may be altered at later stages. (iii) It is indicated for those drugs with narrow therapeutic range like lithium, cyclosporine and amino glycoside antibiotics. (iv) Monitoring is indicated when clinical observations are insufficient to optimize drug dosage. (v) It is indicated to provide information on efficacy, compliance, drug-drug interactions, avoidance of toxicity and therapy cessation monitoring. ${ }^{16,17}$

\section{Benefits to Physician}

(i) By using empiric drug dosage regimen, physician can design dosage regimen for successful therapeutic monitoring services.(ii) It provides confidence in safe and effective dosing.(iii) It is also cost effective for sustained use of drugs like aminoglycoside antibiotics. (iv) Monitoring encourages professional interactions between physician and pharmacist. (v) It is useful to evaluate medication with dosing modifications in patients with renal failure, e.g. selection of antibiotic using empiric therapy after culture and sensitivity test. (vi) Using monitoring, dosing schedule can be developed to optimize initial therapy. (vii) The cost benefit ratio can be improved by performing monitoring. ${ }^{15}$

\section{PRIORI AND POST-PRIORI MONITORING}

Priori monitoring is performed in order to determine initial dosage regimen based on clinical endpoint. The results of PK/PD relationship are useful to identify subpopulations of patients with different dosage requirements. In post-priori monitoring, the data on pre-analytical, analytical and post-analytical phases is generated. The PK monitoring in post-priori TDM is specific, accurate, precise and timely determination of the active and / or toxic forms of drugs in biological samples collected at appropriate time. The PD monitoring in post priori TDM, measurement of biological parameters is as a surrogate or end point marker effect, e.g. plasma concentration of endogenous substances, enzyme activity and gene expression.

\section{LIMITATION OF DRUG MONITORING}

(i) Monitoring of therapeutic drugs is expensive in terms of equipments, supplies, technical experts to obtain concentration in biological fluid, to interpret and investment in research data collection etc. (ii). Only those drugs with broad therapeutic range are benefited by the monitoring. (iii) The data on population of males of $\mathrm{CHF}$ younger than 50 years with normal value of renal cardiac function is available. However, the values are different for female patients older than 70 years suffering from $\mathrm{CHF}$ that cannot be interpreted from the available data on normal values. ${ }^{16}$ (iv) For certain drugs, values of serum concentration are less justifiable than the values of blood pressure measurements and blood coagulation. (v) The data on serum concentration is not required for the drugs with broad therapeutic range.

\section{APPLICATIONS OF DRUG MONITORING}

(i) Monitoring of certain drugs theophylline, valproic acid, methotrexate is associated with the increased efficacy and decreased incidences and side effects. (ii) Monitoring determines the safety of the dosage regimen. If the serum concentration is outside the therapeutic range, 
drug concentration measurements are not required. As response to the drug such as amino-glycoside antibiotics, varies from patient to patient, precision is not maintained due to empiric dosing. (iii) Appropriate interpretation of drug concentration measurements is used in the monitoring for dosage adjustment that minimizes the proportion of patient with serum concentration outside the therapeutic range. (iv) Monitoring encourages professional interactions between members of the health teams such as physician and pharmacist. (v) It provides an opportunity for clinical pharmacist to excel in therapeutic challenges and to work as a vital segment of health team in the area of pharmaco therapeutics. (vi) It provides additional input in pharmacotherapy. For example a) evaluation of medication by dosing modification in patients with impaired renal function. b) Selection of antibiotic e.g. aminoglycoside antibiotics by empiric dosing or culture and sensitivity testing. (vii) It is used to develop dosing protocol which will avoid non-optimum initial therapy.

\section{SUMMARY}

To summarise, the patient response to the life prolonging therapeutic drugs will be improved by monitoring measurements. At the same time, it will reduce substantially drug induced adverse reactions. It was shown that the outcome of drug monitoring services used to obtain clinical pharmacokinetic data, was fewer adverse reactions, shorter intensive care and decrease in overall stay in the hospital. The drug monitoring measurements include three components, structural, process and outcome measures. It is formed of basic infrastructure, drug concentration monitoring methods and implications of results. The monitoring requires combined utilization of pharmaceutical, pharmacokinetic and pharmacodynamics techniques. It is not only measurement of drug concentration and therapeutic range; it also involves the development of safe and effective therapy, dosage individualization, identification of problem with compliance and development of the most effective dosage regimen.

\section{REFERENCES}

1. Aronson JK, Hardman $M . A B C$ of monitoring drug therapy increasing plasma drug concentration. BMJ. 1992;305(6861):1078-80. http://dx.doi.org/10.1136/ bmj.305.6861.1078.

2. Schumacher GE. Editor. Therapeutic drug monitoring. Publisher Pharmamed Press. Appleton and Lange, Medical, 1995.

3. Cristodorescu R, Deutsch G, Dragon S. Clinical utility of plasma digoxin measurement.MedInterne. 1989;27(1):25-32. PMid:2749155.

4. Zhou W, Jacq R, Aigrain E. Principles of Therapeutic drug monitoring. Handbook Exptl Pharmacol.2011;205:77-90. http://dx.doi.org/10.1007/978-3642-20195-0_3; PMid:21882106.

5. Gross AS. Best practices in therapeutic drug monitoring. BrJClinPharmacol.2 001;52(Suppl.1):5S-10S. http://dx.doi.org/10.1046/j.1365-2125.2001.00770.x: http://dx.doi.org/10.1111/j.1365-2125.2001.00770.x PMid:11564048 PMCid:PMC2014621.

6. McInnes GT. The value of therapeutic drug monitoring to the practicing physician. Br J Clin Pharmacol. 1989;27(3):281-4. http://dx.doi.org/10.1111/j.1365-2125.1989. tb05365.x; PMid:2719892: PMCid:PMC1379823.

7. Reynolds DJ, Aronson JK. ABC of monitoring drug therapy : making the most of plasma drug concentration measurements. BMJ.1993;306(6861):48-51. http:// dx.doi.org/10.1136/bmj.306.6869.48 ; PMid:8435581 PMCid:PMC1676398.

8. Suthakaran C, Adithan C. Therapeutic drug monitoring -concept, methodology, clinical applications and limitations. Ch.7, Health Administrator, 2006;1:22-26.

9. Lawson G, Cocks E, Tanna S. Quantitative determination of atenelol in dried blood spot samples by LC-HRMS or potential method for assessing medication adherence. JChromatogr B Analyst Technol Biomed Life Sci. 2012;15:817:72-9.

10. Millership JS. Micro assay of drugs and modern measurement technique. Pediatric Anaesth. 2011;21(3):197-205. http://dx.doi.org/10.1111/j.14609592.2011.03535.x ; PMid:21320232.

11. Mullet WM. Determination of drugs in biological fluid by direct injection of samples of liquid chromatographic analysis. JBiolChem Methods. 2007;70(2):263-73. http://dx.doi.org/10.1016/j.jbbm.2006.10.001; PMid:17113152.

12. Manicke NE, Abu-Rabie P, Spooner N, Ouyang Z, Cooks RG. Quantitative analysis of therapeutic drugs in dried blood spot samples by paper spray mass spectrometry: an avenue to therapeutic drug monitoring.JAmSocMass Spectrum. 2011;22(9):1501-7. http://dx.doi.org/10.1007/s13361-011-0177-x .

13. Buchner F,Tonkin A. The clinician and therapeutic drug monitoring in 1990s. MedJAust.1993;158(6):422-6.

14. Vozeh S. Cost effectiveness of therapeutic drug monitoring1993. Clin Pharmacokin. 1987;13:131-40. http://dx.doi.org/10.2165/00003088-19871303000001.

15. Kang LS, Lee MM. Overview of Therapeutic drug monitoring. Korean JIntern Med. 2009;24(1):1-10. http://dx.doi.org/10.3904/kjim.2009.24.1.1; PMid:19270474 PMCid:PMC2687654.

16. Barr JT, Schumacher GE.Outcome assessment of therapeutic drug monitoring. In Schumacher GE, editor, Therapeutic drug monitoring1995; East Appleton \& Lange, 1995;191-236. 